\title{
There are Finitely Many Triangle-Free Distance-Regular Graphs with Degree 8, 9 or 10
}

J.H. KOOLEN*

jhk@euclid.postech.ac.kr

Division of Applied Mathematics, KAIST 373-1 Kusongdong, Yusongku Deajon, 305701 Korea

V. MOULTON ${ }^{\dagger}$

vincent.moulton@1cb.uu.se

The Linnaeus Centre for Bioinformatics, Uppsala University, BMC, Box 598, 75124 Uppsala, Sweden

Received August 19, 2002; Revised March 19, 2003; Accepted April 18, 2003

Abstract. In this paper we prove that there are finitely many triangle-free distance-regular graphs with degree 8,9 or 10 .

Keywords: distance-regular graphs, Bannai-Ito conjecture

\section{Introduction}

In [1] Bannai and Ito conjectured that there finitely many distance-regular graphs with a fixed degree at least 3, and in the series of papers [2-5], they showed that their conjecture held for degrees 3 and 4. In [7], we showed that there are finitely many distance-regular graphs with degree 5,6 , or 7 . Here we extend this result, showing that there are finitely many triangle-free distance-regular graphs with degree 8,9 or 10 .

Suppose that $k$ is an integer with $k \geq 3$ and that $\Gamma$ is a distance-regular graph with degree $k$, diameter $d \geq 2$ and intersection numbers $a_{i}, b_{i}, c_{i}, 0 \leq i \leq d$. We call the sequence $\left(\left(c_{i}, a_{i}, b_{i}\right) \mid 1 \leq i \leq d-1\right)$ the tridiagonal sequence of $\Gamma$. Given integers $a \geq 0$ and $b, c \geq 1$ with $a+b+c=k$, we define

$$
l_{(c, a, b)}=l_{(c, a, b)}(\Gamma):=\mid\left\{i \mid 1 \leq i \leq d-1 \text { and }\left(c_{i}, a_{i}, b_{i}\right)=(c, a, b)\right\} \mid,
$$

and put

$$
\mathrm{h}=\mathrm{h}_{\Gamma}:=l_{\left(1, a_{1}, b_{1}\right)} \text { and } \mathrm{t}=\mathrm{t}_{\Gamma}:=l_{\left(b_{1}, a_{1}, 1\right)} .
$$

Note that the first $\mathrm{h}$ terms of the tridiagonal sequence of $\Gamma$ are all equal to $\left(1, a_{1}, b_{1}\right)$ and, if $t>0$, then the last $t$ terms of this sequence are all equal to $\left(b_{1}, a_{1}, 1\right)$. In this paper we will prove the following theorem.

*The author thanks the Com2MaC-KOSEF for its support.

${ }^{\dagger}$ The author thanks the Swedish Research Council (VR) for its support. 
Theorem 1.1 Suppose $k \geq 3$ is an integer. There exists a real number $\alpha>0$, depending only on $k$ so that there are finitely many triangle-free distance-regular graphs $\Gamma$ with degree $k$, and diameter $d$ satisfying

$$
d-\left(\mathrm{h}_{\Gamma}+\mathrm{t}_{\Gamma}\right) \leq \alpha \mathrm{h}_{\Gamma} .
$$

Remark 1.2 (i) In the proof of Theorem 1 it can be seen that $\alpha$ tends to zero as $k$ tends to $\infty$. We would like to show that the theorem still holds in case $\alpha$ does not depend on $k$ (which would follow if, for example, the second largest eigenvalue of a distance-regular graph $\Gamma$ were always large enough).

(ii) If $\alpha \mathrm{h}_{\Gamma}$ is replaced by a constant in Theorem 1.1, then we obtain a result of Bannai and Ito [5]. However, we use their result in our proof of Theorem 1.1.

To describe the consequences of Theorem 1.1 we require some further definitions. Put

$$
V_{k}:=\left\{(c, a, b) \in \mathbf{Z}^{3} \mid a \geq 0 \text { and } b, c \geq 1 \text { and } a+b+c=k\right\}
$$

and

$$
V_{k}^{*}:=V_{k} \backslash\{(1,0, k-1),(k-1,0,1)\} .
$$

For any $(c, a, b) \in V_{k}$ define the open real interval

$$
I_{(c, a, b)}:=(a-2 \sqrt{b c}, a+2 \sqrt{b c}) .
$$

We say that a subset $\Pi \subseteq V_{k}^{*}$ satisfies the interval intersection property (IIP) if

$$
\bigcap_{(c, a, b) \in \Pi} I_{(c, a, b)} \neq \varnothing
$$

(so that, in particular, the empty set satisfies the interval intersection property).

Now, for a distance regular graph $\Gamma$ as above, put

$$
\Omega^{*}(\Gamma):=\left\{\left(c_{i}, a_{i}, b_{i}\right) \mid 1 \leq i \leq d-1\right\} \backslash\left\{\left(1, a_{1}, b_{1}\right),\left(b_{1}, a_{1}, 1\right)\right\} .
$$

In [7, Theorem 7.2] we showed that in case $\Pi \subseteq V_{k}^{*}$ satisfies (IIP) and $\epsilon$ is any positive real number, there are finitely many triangle-free distance-regular graphs with degree $k$, diameter $d$, and $\Omega^{*}(\Gamma) \subseteq \Pi$ for which

$$
d-(\mathrm{h}+\mathrm{t}) \geq \epsilon \mathrm{h}
$$

holds. Thus, as a consequence of Theorem 1.1 we obtain the following result.

Theorem 1.3 Suppose $k \geq 3$ is an integer and $\Pi \subseteq V_{k}^{*}$ satisfies (IIP). Then there are finitely many triangle-free distance-regular graphs $\Gamma$ with degree $k$ and $\Omega^{*}(\Gamma) \subseteq \Pi$. 
Remark 1.4 Note that the set

$$
\Pi^{\prime}:=\{(c, 0, k-c) \mid c=1,2, \ldots, k-1\}
$$

satisfies (IIP) since $0 \in I_{(c, 0, k-c)}$ for all $c=1,2, \ldots, k-1$. Since for any bipartite distanceregular graph $\Gamma$ of degree $k$ we have $\Omega^{*}(\Gamma) \subseteq \Pi^{\prime}$, it follows by Theorem 1.3 that there are finitely many bipartite distance-regular graphs with degree $k \geq 3$. This result was established by Bannai and Ito in [4]. However, the techniques that we adopt in this paper may be used to provide an improvement on their upper bound for the diameter of a bipartite distance-regular graph for fixed degree $k$.

In [7, Lemma 3.1], we showed that the set $V_{k}^{*}$ satisfies (IIP) if and only if $3 \leq k \leq 10$. In view of this and the last theorem we obtain the main result of this paper.

Corollary 1.5 There are finitely many triangle-free distance-regular graphs with degree 8,9 , or 10 .

We close this section by briefly describing the contents of this paper. In Section 2 we recall some facts concerning distance-regular graphs and also provide bounds for the multiplicities of the eigenvalues of a distance-regular graph. Using these bounds together with a polynomial that we study in Section 3, we prove Theorem 1.1 in Section 4.

\section{Multiplicities of eigenvalues}

We begin this section by recalling some facts concerning distance-regular graphs (for more details see [6]). Suppose that $\Gamma$ is a connected graph. The distance $d(u, v)$ between any two vertices $u, v$ in the vertex set $V \Gamma$ of $\Gamma$ is the length of a shortest path between $u$ and $v$ in $\Gamma$. For any $v \in V \Gamma$, define $\Gamma_{i}(v)$ to be the set of vertices in $\Gamma$ at distance precisely $i$ from $v$, where $i$ is any non-negative integer not exceeding the diameter of $\Gamma$. In addition, define $\Gamma_{-1}(v)=\Gamma_{d+1}(v):=\emptyset$. Following [6], we call a connected graph $\Gamma$ with diameter $d$ distance-regular if there are integers $b_{i}, c_{i}, 0 \leq i \leq d$, such that for any two vertices $u, v \in V \Gamma$ at distance $i=d(u, v)$, there are precisely $c_{i}$ neighbors of $v$ in $\Gamma_{i-1}(u)$ and $b_{i}$ neighbors of $v$ in $\Gamma_{i+1}(u)$. In particular, $\Gamma$ is regular with degree $k:=b_{0}$. For $i=0, \ldots, d$, set

$$
a_{i}:=k-b_{i}-c_{i},
$$

which equals the number of neighbors of $v$ in $\Gamma_{i}(u)$ where $d(u, v)=i$. The numbers $c_{i}, b_{i}, a_{i}$ are called the intersection numbers of $\Gamma$. Clearly $b_{d}=c_{0}=a_{0}=0$ and $c_{1}=1$ and, as is shown in [6, Section 4.1], $\Gamma_{i}(u)$ contains $k_{i}$ elements, where

$$
k_{0}:=1, \quad k_{1}:=k, \quad k_{i+1}:=k_{i} b_{i} / c_{i+1}, \quad i=0, \ldots, d-1 .
$$


Moreover, as is shown in [6, Proposition 4.1.6], the following inequalities must hold

$$
k=b_{0}>b_{1} \geq b_{2} \geq \cdots \geq b_{d-1}>b_{d}=0 \text { and } 1=c_{1} \leq c_{2} \leq \cdots \leq c_{d} \leq k
$$

Note that a distance-regular graph $\Gamma$ is triangle-free (i.e. contains no 3-cycles) if and only if $a_{1}=0$.

Now, suppose that $\Gamma$ is a distance-regular graph with degree $k$, diameter $d$ and intersection numbers $a_{i}, b_{i}, c_{i}, 0 \leq i \leq d$. Recall that if $\theta$ is an eigenvalue of $\Gamma$, then $\theta \in[-k, k]$. The standard sequence $\left(u_{i}=u_{i}(\theta) \mid 0 \leq i \leq d\right)$ associated to each eigenvalue $\theta$ of $\Gamma$ (i.e. eigenvalue of the adjacency matrix of $\Gamma$ ) is defined recusively by the equations

$$
u_{0}=1, u_{1}=\theta / k, b_{i} u_{i+1}-\left(\theta-a_{i}\right) u_{i}+c_{i} u_{i-1}=0 \quad \text { for } i=1,2, \ldots, d-1 .
$$

It is well-known, see e.g. [6, Theorem 4.1.4], that the multiplicity $m(\theta)$ of any eigenvalue $\theta$ of $\Gamma$ is given by $m(\theta)=\frac{|V \Gamma|}{M(\theta)}$ where

$$
M(\theta)=\sum_{i=0}^{d} k_{i} u_{i}(\theta)^{2} .
$$

Although the following result was shown by Bannai and Ito in [4], we give its proof for the reader's convenience.

Lemma 2.1 Suppose that $\Gamma$ is a distance-regular graph with degree $k \geq 3$ and diameter $d \geq 2$. Suppose also that $\theta$ is an eigenvalue of $\Gamma$ and that $\left(u_{i} \mid 0 \leq i \leq d\right)$ is the standard sequence corresponding to $\theta$. Then

$$
\frac{1}{3 k} \max \left\{\left|u_{i}\right|,\left|u_{i+1}\right|\right\} \leq \max \left\{\left|u_{i-1}\right|,\left|u_{i}\right|\right\} \leq 3 k \max \left\{\left|u_{i}\right|,\left|u_{i+1}\right|\right\}
$$

holds for $i=1, \ldots, d-1$.

Proof: $\quad$ Since the numbers $u_{i}, i=0,1, \ldots, d$, satisfy

$$
c_{i} u_{i-1}+a_{i} u_{i}+b_{i} u_{i+1}=\theta u_{i}, \quad i=1,2, \ldots, d-1,
$$

and $b_{i} \geq 1$ for $i=1, \ldots, d-1$, it follows that

$$
u_{i+1}=\frac{-c_{i} u_{i-1}+\left(\theta-a_{i}\right) u_{i}}{b_{i}}, \quad i=1,2, \ldots, d-1,
$$

holds. Thus, since $0 \leq a_{i}, c_{i}$ for $i=1, \ldots, d-1$ and $|\theta| \leq k$, we have

$$
\left|u_{i+1}\right| \leq k\left|u_{i-1}\right|+2 k\left|u_{i}\right|, \quad i=1,2, \ldots, d-1 .
$$

Now suppose $\max \left\{\left|u_{i-1}\right|,\left|u_{i}\right|\right\}=\left|u_{i}\right|, i=1,2, \ldots, d-1$. Then

$$
\left|u_{i+1}\right| \leq k\left|u_{i-1}\right|+2 k\left|u_{i}\right| \leq 3 k\left|u_{i}\right|,
$$


and from this it easily follows that $\frac{1}{3 k} \max \left\{\left|u_{i}\right|,\left|u_{i+1}\right|\right\} \leq\left|u_{i}\right|$ holds. Moreover, if $\max \left\{\left|u_{i-1}\right|\right.$, $\left.\left|u_{i}\right|\right\}=\left|u_{i-1}\right|, i=1,2, \ldots, d-1$, then

$$
\left|u_{i+1}\right| \leq k\left|u_{i-1}\right|+2 k\left|u_{i}\right| \leq 3 k\left|u_{i-1}\right|
$$

from which it follows that $\frac{1}{3 k} \max \left\{\left|u_{i}\right|,\left|u_{i+1}\right|\right\} \leq\left|u_{i-1}\right|$ holds. Hence

$$
\frac{1}{3 k} \max \left\{\left|u_{i}\right|,\left|u_{i+1}\right|\right\} \leq \max \left\{\left|u_{i-1}\right|,\left|u_{i}\right|\right\}
$$

holds, which is the right-hand inequality in the statement of the lemma.

The proof of the left-hand inequality in the statement of the lemma is similar (simply interchange the roles of $u_{i+1}$ and $u_{i-1}$ ).

Define $\rho_{1}(\theta)=\rho_{1}\left(\rho_{2}(\theta)=\rho_{2}\right)$ to be the largest (smallest) root in absolute value of the quadratic equation

$$
(k-1) x^{2}-\theta x+1
$$

Note that $\rho_{1}(\theta)$ is an increasing function of $\theta$ in the interval $(\sqrt{2 k-1}, \infty)$.

Proposition 2.2 Suppose that $\alpha$ and $\epsilon$ are positive real numbers and that $k \geq 3$ is an integer. If $\theta$ is an eigenvalue of a triangle-free distance-regular graph $\Gamma$ with degree $k$ and diameter $d$ satisfying $d-(\mathrm{h}+\mathrm{t}) \leq \alpha \mathrm{h}$, then there are constants $A, B$ depending only on $\alpha, \epsilon$ and $k$ (and not $\theta$ ) so that the following statements hold:

(i) If $|\theta|>2 \sqrt{k-1}+\epsilon$, then

$$
\rho_{1}(\theta)^{2 \mathrm{~h}}(k-1)^{\mathrm{h}} \leq M(\theta) \leq A\left(9 k^{3}\right)^{\alpha \mathrm{h}} \rho_{1}(\theta)^{2 \mathrm{~h}}(k-1)^{\mathrm{h}} .
$$

(ii) If $|\theta|<2 \sqrt{k-1}-\epsilon$, then

$$
M(\theta) \leq B \mathrm{~h}\left(9 k^{3}\right)^{\alpha \mathrm{h}} .
$$

Proof: (i) Suppose $\theta>2 \sqrt{k-1}+\epsilon$.

Claim 1 There are positive constants $C_{1}, C_{2}$, and $C_{3}$ depending only on $k$ and $\epsilon$ so that

$$
C_{1} \rho_{1}^{2 \mathrm{~h}}(k-1)^{\mathrm{h}} \leq \sum_{i=0}^{\mathrm{h}} k_{i} u_{i}^{2} \leq C_{2} \rho_{1}^{2 \mathrm{~h}}(k-1)^{\mathrm{h}}
$$

and

$$
\rho_{1}^{2 \mathrm{~h}}(k-1)^{\mathrm{h}} \leq \max \left\{k_{\mathrm{h}} u_{\mathrm{h}}^{2}, k_{\mathrm{h}+1} u_{\mathrm{h}+1}^{2}\right\} \leq C_{3} \rho_{1}^{2 \mathrm{~h}}(k-1)^{\mathrm{h}} .
$$


Proof of Claim 1: By definition of $\mathrm{h}$, for each $i=1,2, \ldots, \mathrm{h}$ we have $\left(c_{i}, a_{i}, b_{i}\right)=$ $(1,0, k-1)$, and hence the first $\mathrm{h}+2$ equations defining the standard sequence for $\theta$ are

$$
u_{0}=1, u_{1}=\theta / k, \quad \text { and } \quad(k-1) u_{i+1}-\theta u_{i}+u_{i-1}=0 \quad \text { for } i=1,2, \ldots, \mathrm{h} .
$$

Since $\theta>2 \sqrt{k-1}+\epsilon$, there is some $\kappa$ (depending on $\epsilon$ ) with $2 \sqrt{k-1}<\kappa<\theta<k$. Hence by [7, Proposition 4.1] it follows that

$$
\rho_{1}^{i} \leq\left|u_{i}\right| \leq \tau \rho_{1}^{i}, \quad i=0,1, \ldots, \mathrm{h}+1,
$$

holds, where

$$
\tau=\tau(\kappa, k):=1+\left(\frac{\kappa-k \rho_{1}(\kappa)}{k\left(\rho_{1}(\kappa)-\rho_{2}(\kappa)\right)}\right) .
$$

Now, by (1) and (2) we have

$$
k_{i}=k(k-1)^{i-1}, 1 \leq i \leq \mathrm{h}, \quad \text { and } \quad k_{\mathrm{h}+1} \leq(k-1) k_{\mathrm{h}} .
$$

Hence

$$
\sum_{i=1}^{\mathrm{h}} k_{i} u_{i}^{2}=k \sum_{i=1}^{\mathrm{h}}(k-1)^{i-1} u_{i}^{2}
$$

and so in view of (5) and (6) we have

$$
\rho_{1}^{2 \mathrm{~h}}(k-1)^{\mathrm{h}}<k_{\mathrm{h}} u_{\mathrm{h}}^{2} \leq \sum_{i=1}^{\mathrm{h}} k_{i} u_{i}^{2} \leq k \rho_{1}^{2} \tau^{2}\left[\frac{1-\rho_{1}^{2 \mathrm{~h}}(k-1)^{\mathrm{h}}}{1-\rho_{1}^{2}(k-1)}\right] .
$$

But $\frac{1}{\sqrt{k-1}}=\rho_{1}(2 \sqrt{k-1})<\rho_{1}(\kappa)<\rho_{1}(\theta)<\rho_{1}(k)=1$, where the first equality follows from simple computation and the subsequent inequalities from the fact that $\rho_{1}$ is an increasing function of $\theta$ on $(2 \sqrt{k-1}, \infty)$. Therefore since $k_{0} u_{0}^{2}=1$ it follows that

$$
\rho_{1}^{2 \mathrm{~h}}(k-1)^{\mathrm{h}} \leq \sum_{i=0}^{\mathrm{h}} k_{i} u_{i}^{2} \leq 1+\rho_{1}^{2 \mathrm{~h}}(k-1)^{\mathrm{h}}\left[\frac{\tau^{2} k}{(k-1)\left(\rho_{1}(\kappa)\right)^{2}-1}\right]
$$

must hold. From this it is straight-forward to check that there are positive constants $C_{1}$ and $C_{2}$ depending only on $k, \kappa$ (and hence on $k, \epsilon$ ) so that (3) holds.

To see that there is a positive constant $C_{3}$ depending only on $k, \epsilon$ so that (4) holds, note that the left-hand inequality follows immediately from (5) and (6), whereas the right-hand inequality can be seen to hold using (5), (6) and $k_{\mathrm{h}+1} \leq(k-1) k_{\mathrm{h}}$. 
Claim 2 There are positive constants $C_{4}, C_{5}$, and $C_{6}$ depending only on $k$ and $\epsilon$ so that

$$
C_{4} \rho_{1}^{2 \mathrm{~h}}(k-1)^{\mathrm{h}} \leq \sum_{i=0}^{d-\mathrm{t}} k_{i} u_{i}^{2} \leq C_{5}\left(9 k^{3}\right)^{\alpha \mathrm{h}} \rho_{1}^{2 \mathrm{~h}}(k-1)^{\mathrm{h}}
$$

and

$$
\max \left\{k_{d-\mathrm{t}-1} u_{d-\mathrm{t}-1}^{2}, k_{d-\mathrm{t}} u_{d-\mathrm{t}}^{2}\right\} \leq C_{6}\left(9 k^{3}\right)^{\alpha \mathrm{h}} \rho_{1}^{2 \mathrm{~h}}(k-1)^{\mathrm{h}} .
$$

Proof of Claim 2: By Lemma 2.1 we have

$$
\sum_{i=\mathrm{h}+1}^{d-\mathrm{t}} k_{i} u_{i}^{2} \leq k_{\mathrm{h}+1} u_{\mathrm{h}+1}^{2} \sum_{j=0}^{d-\mathrm{t}-\mathrm{h}-1}\left((3 k)^{2}(k-1)\right)^{j} \leq k_{\mathrm{h}+1} u_{\mathrm{h}+1}^{2}\left(9 k^{3}\right)^{\alpha \mathrm{h}} .
$$

The existence of positive constants $C_{4}, C_{5}$ so that the first two inequalities in the statement of Claim 2 hold now follows from Claim 1 . The existence of a constant $C_{6}$ so that the last inequality holds follows from Claim 1, Lemma 2.1 and (8).

We now complete the proof of (i) in case $\theta>2 \sqrt{k-1}+\epsilon$ holds. If $t=0$, then (i) follows directly from Claim 2 since then

$$
M(\theta)=\sum_{i=0}^{d} k_{i} u_{i}^{2}=\sum_{i=0}^{d-1} k_{i} u_{i}^{2}+k_{d} u_{d}^{2}
$$

In case $t>0$, note first that by [7, Lemma 2.1] we have $a_{d}=0$. By definition of $t$, for each $i=d-\mathrm{t}, \ldots, d-1$ we have $\left(c_{i}, a_{i}, b_{i}\right)=(k-1,0,1)$, and so the equations defining the standard sequence for $\theta$ for $d-\mathrm{t} \leq i \leq d$ can be written as

$$
u_{d-1}=(\theta / k) u_{d} \text { and }(k-1) u_{d-i-1}-\theta u_{d-i}+u_{d-i+1}=0, \quad i=1,2, \ldots, \mathrm{t} .
$$

Using [7, Proposition 4.1] it is thus straight-forward to see that

$$
\left|u_{d}\right| \rho_{1}^{i} \leq\left|u_{d-i}\right| \leq\left|u_{d}\right| \tau \rho_{1}^{i}, \quad i=1, \ldots, \mathrm{t}+1
$$

must hold.

Hence, we see-in a similar way to the way in which we showed that (7) follows from (5)-that

$$
\begin{aligned}
\rho_{1}^{2 \mathrm{~h}}(k-1)^{\mathrm{h}} k_{d} u_{d}^{2} & \leq \sum_{i=d-\mathrm{t}}^{d} k_{i} u_{i}^{2} \\
& \leq k \rho_{1}^{2} k_{d} u_{d}^{2}\left(1+\rho_{1}^{2 \mathrm{~h}}(k-1)^{\mathrm{h}}\left[\frac{\tau^{2} k}{(k-1)\left(\rho_{1}(\kappa)\right)^{2}-1}\right]\right)
\end{aligned}
$$


must hold. The case where $t>0$ hlds now follows in a straight-forward fashion from (9), (10) and Claim 2.

To see that (i) holds in case $\theta<-2 \sqrt{k-1}-\epsilon$ note that since $\rho_{1}(\theta)=-\rho_{1}(-\theta)$, $u_{i}(\theta)=-u_{i}(-\theta), 0 \leq i \leq \mathrm{h}$ and $u_{d-i}(\theta)=u_{i}(\theta) u_{d}(\theta)$ for $0 \leq i \leq \mathrm{t}$, we have

$$
\sum_{i=0}^{\mathrm{h}} k_{i} u_{i}(\theta)^{2}=\sum_{i=0}^{\mathrm{h}} k_{i} u_{i}(-\theta)^{2}
$$

and

$$
\sum_{i=d-\mathrm{t}}^{d} k_{i} u_{i}(\theta)^{2}=k_{d} u_{d}(\theta)^{2} \sum_{i=0}^{\mathrm{t}} k_{i} u_{i}(\theta)^{2}=k_{d} u_{d}(\theta)^{2} \sum_{i=0}^{\mathrm{t}} k_{i} u_{i}(-\theta)^{2} .
$$

It is now straight-forward to complete the proof of (i) using similar claims and arguments to those just given above to show that (i) holds in case $\theta>2 \sqrt{k-1}+\epsilon$.

(ii) Assume $|\theta|<2 \sqrt{k-1}-\epsilon$.

Claim 3 There are positive constants $C_{1}, C_{2}$ depending only on $k$ and $\epsilon$ with

$$
\sum_{i=0}^{\mathrm{h}} k_{i} u_{i}^{2} \leq C_{1} \mathrm{~h}
$$

and

$$
\max \left\{k_{\mathrm{h}} u_{\mathrm{h}}^{2}, k_{\mathrm{h}+1} u_{\mathrm{h}+1}^{2}\right\} \leq C_{2} .
$$

Proof of Claim 3: By [7, Proposition 4.2] we have

$$
\sum_{i=0}^{\mathrm{h}}(k-1)^{i} u_{i}^{2} \leq C_{1}^{\prime} \max \left\{u_{0}^{2}, u_{1}^{2}\right\}(\mathrm{h}+1)
$$

where $C_{1}^{\prime}$ is a positive constant depending only on $k$ and $\epsilon$. But then using (6) and $u_{0}=1$ it is now straight-forward to show that there exists a positive constant $C_{1}$ for which the first inequality in Claim 3 holds.

Now, by [7, Proposition 4.2], we have

$$
(k-1)^{\mathrm{h}} \max \left\{u_{\mathrm{h}}^{2}, u_{\mathrm{h}+1}^{2}\right\} \leq C_{2}^{\prime} \max \left\{u_{0}^{2}, u_{1}^{2}\right\},
$$

where $C_{2}^{\prime}$ is a positive constant depending only on $k$ and $\epsilon$. The existence of a positive constant $C_{2}$ for which the second inequality in Claim 3 holds follows in view of this and (6).

Claim 4 There are positive constants $C_{4}, C_{5}$ depending only on $k$ and $\epsilon$ so that

$$
\sum_{i=0}^{d-\mathrm{t}} k_{i} u_{i}^{2} \leq C_{4} \mathrm{~h}\left(9 k^{3}\right)^{\alpha \mathrm{h}}
$$


and

$$
\max \left\{k_{d-\mathrm{t}-1} u_{d-\mathrm{t}-1}^{2}, k_{d-\mathrm{t}} u_{d-\mathrm{t}}^{2}\right\} \leq C_{5}\left(9 k^{3}\right)^{\alpha \mathrm{h}} .
$$

Proof of Claim 4: The existence of a positive constant $C_{4}$ so that the first inequality holds follows from Claim 3 and (8). The existence of a positive constant $C_{5}$ so that the second inequality holds follows from Claim 3 and Lemma 2.1.

We now complete the proof of (ii). If $t=0$, then (ii) follows immediately from Claim 4 . If $t>0$, then first note that

$$
\sum_{i=d-\mathrm{t}}^{d} k_{i} u_{i}^{2}=k_{d} u_{d}\left(1+\sum_{i=1}^{\mathrm{t}} k(k-1)^{i-1} u_{i}^{2}\right)
$$

holds. Now, in a similar way to the way in which we proved Claim 3, it is straight-forward to show that there exists a positive constant $C_{1}^{\prime}$ depending only on $k$ and $\epsilon$ with

$$
1+\sum_{i=1}^{\mathrm{t}} k(k-1)^{i-1} u_{i}^{2} \leq C_{1}^{\prime} \mathrm{t} .
$$

Since $u_{d-\mathrm{t}-1}, u_{d-\mathrm{t}}, \ldots, u_{d}$ satisfy

$$
(k-1) u_{i-1}+\theta u_{i}+u_{i+1} \quad i=d-\mathrm{t}, \ldots, d-1,
$$

it follows by [7, Proposition 4.2] that there exists a positive constant $C_{2}^{\prime}$ depending only on $k, \epsilon$ with

$$
(k-1)^{\mathrm{t}} \max \left\{u_{d-\mathrm{t}-1}^{2}, u_{d-\mathrm{t}}^{2}\right\} \leq C_{2}^{\prime} \max \left\{u_{d-1}^{2}, u_{d}^{2}\right\} .
$$

Since $k_{d-i}=k_{d} k(k-1)^{i-1}$ for $i=1, \ldots, \mathrm{t}$, this immediately implies the existence of a positive constant $C_{3}^{\prime}$ depending only on $k, \epsilon$ with

$$
\max \left\{k_{d-\mathrm{t}-1} u_{d-\mathrm{t}-1}^{2}, k_{d-\mathrm{t}} u_{d-\mathrm{t}}^{2}\right\} \leq C_{3}^{\prime} k_{d} u_{d}^{2} .
$$

Using this and Claim 4, it is now straight-forward to see that (ii) holds.

\section{A useful polynomial}

Suppose that $k \geq 3$ is an integer. Put

$$
\begin{aligned}
P(x)= & \prod_{(c, a, b) \in V_{k}, c \leq b}(x-a-2 \sqrt{b c})(x+a-2 \sqrt{b c})(x-a+2 \sqrt{b c}) \\
& \times(x+a+2 \sqrt{b c}) .
\end{aligned}
$$


It is straight-forward to verify that $P$ has the following properties:

(i) $P \not \equiv 0$.

(ii) $P$ has integral coefficients.

(iii) If $(c, a, b) \in V_{k}$, then $a+2 \sqrt{b c}$ and $a-2 \sqrt{b c}$ are roots of $P$.

(iv) $P$ is even (i.e. $P(x)=P(-x)$ for $x \in \mathbb{R}$ ).

Now suppose

$$
\beta:=\frac{(2 \sqrt{k-1})+(1+2 \sqrt{k-2})}{2}=\frac{1}{2}+\sqrt{k-1}+\sqrt{k-2} .
$$

Since $k \geq 3$, it follows that $\beta>k$. Moreover, in [7, Lemma 3.1] it is shown that

$$
\min \left\{a+2 \sqrt{b c} \mid(c, a, b) \in V_{k}^{*}\right\}=1+2 \sqrt{k-2}
$$

holds, from which it easily follows that $a+2 \sqrt{b c}>\beta$ holds for all $(c, a, b) \in V_{k}^{*}$.

Now define

$$
S_{1 / 2}:=\{x \in[\beta, k]|0<| P(x) \mid<1 / 2\} .
$$

Clearly $S_{1 / 2}$ consists of a collection of disjoint open intervals and $a+2 \sqrt{b c} \notin S_{1 / 2}$ for all $(c, a, b) \in V_{k}^{*}$. Put

$$
S_{1}:=\{x \in[-k, k]|| P(x) \mid \geq 1\} .
$$

We conclude this section with a lemma that follows easily from the facts that $P$ is continuous and even.

Lemma 3.1 There exists a real number $\gamma>0$ depending on $k$ such that

$$
|| x|-| y|| \geq \gamma
$$

holds for all $x \in S_{1}$ and $y \in S_{1 / 2}$.

\section{Proof of Theorem 1.1}

We first prove three claims, from which the theorem will follow.

Claim 1 Suppose $\Gamma$ is a triangle-free distance-regular graph with degree $k$ and diameter $d$. There exists a constant $M \geq 0$ depending only on $k$ so that if $d-(\mathrm{h}+\mathrm{t})>M$, then $\Gamma$ has an eigenvalue $\theta$ with $\theta \in S_{1 / 2}$. 
Proof of Claim 1: Suppose $(c, a, b) \in V_{k}^{*}$. If $l:=l_{(c, a, b)} \geq 3$, then by [7, Theorem 6.2 (ii)] $\Gamma$ has an eigenvalue $\theta$ with

$$
a+2 \sqrt{b c} \cos \left(\frac{j \pi}{l+1}\right) \leq \theta \leq a+2 \sqrt{b c} \cos \left(\frac{(j-2) \pi}{l+1}\right),
$$

where $3 \leq j \leq l$. As noted above, $S_{1 / 2}$ consists of a disjoint union of non-empty open intervals. Suppose $(\tau, a+2 \sqrt{b c})$ with $\tau$ a real number is one of these intervals, which we can assume since $a+2 \sqrt{b c}$ is a root of $P$. Since

$$
\lim _{l \rightarrow \infty}\left[a+2 \sqrt{b c} \cos \left(\frac{j \pi}{l+1}\right)\right]=a+2 \sqrt{b c},
$$

there must exist some $L=L(c, a, b) \geq 3$ depending only on $(c, a, b)$ so that

$$
\left(a+2 \sqrt{b c} \cos \left(\frac{j \pi}{L+1}\right), a+2 \sqrt{b c}\right) \subseteq S_{1 / 2}
$$

holds. Thus, by putting

$$
M=\sum_{(c, a, b) \in V_{k}^{*}} L(c, a, b)
$$

we see that if $d \geq \mathrm{h}+\mathrm{t}+M+1$, then $\Gamma$ has an eigenvalue $\theta$ with $\theta \in S_{1 / 2}$. Moreover, $M$ clearly only depends on $k$. This concludes the proof of Claim 1.

Claim 2 Suppose $\Gamma$ is a triangle-free distance-regular graph with degree $k$. If $\Gamma$ has an eigenvalue $\theta \in S_{1 / 2}$, then $\theta$ has an algebraic conjugate $\theta^{\prime}$ with $\theta^{\prime} \in S_{1}$.

Proof of Claim 2: Since $P$ has integer coefficients and any eigenvalue of $\Gamma$ is an algebraic integer, it follows that

$$
\prod_{\eta \text { algebraic conjugate of } \theta} P(\eta)
$$

is an integer. Moreover, this is a non-zero integer since $P$ is a polynomial with integer coefficients and leading coefficient one and $P(\eta) \neq 0$ for $\eta$ any algebraic conjugate of $\theta$ (as $P(\theta) \neq 0$ ). Hence, $\theta$ must have some algebraic conjugate $\theta^{\prime}$ with $\left|P\left(\theta^{\prime}\right)\right| \geq 1$, that is, $\theta^{\prime} \in S_{1}$.

Claim 3 There exist constants $\alpha, R>0$, each depending only on $k$, so that if $\Gamma$ is a triangle-free distance-regular graph with degree $k$, diameter $d$, some eigenvalue in $S_{1 / 2}$, and $d-(\mathrm{h}+\mathrm{t}) \leq \alpha \mathrm{h}$, then $\mathrm{h} \leq R$.

Proof of Claim 3: Suppose $\theta \in S_{1 / 2}$ is an eigenvalue of $\Gamma$. By Claim 2, $\theta$ has an algebraic conjugate $\theta^{\prime} \in S_{1}$ so that, in particular, $M(\theta)=M\left(\theta^{\prime}\right)$. 
Note that by Lemma 3.1 there is some positive real number $\gamma$ with ||$\theta^{\prime}|-| \theta|| \geq \gamma$, and by definition of $S_{1 / 2},|\theta| \geq \beta$, and hence $\left|\rho_{1}(\theta)\right| \geq \rho_{1}(\beta)>1 / \sqrt{k-1}$.

We now consider seperately the two cases when $\theta^{\prime}$ is contained in the closed interval $[-2 \sqrt{k-1}, 2 \sqrt{k-1}]$ and when it is not.

Case 1. $\theta^{\prime} \in[-2 \sqrt{k-1}, 2 \sqrt{k-1}]$.

Since $\theta^{\prime} \in[-2 \sqrt{k-1}, 2 \sqrt{k-1}]$ it follows that $\left|\rho_{1}\left(\theta^{\prime}\right)\right|=1 / \sqrt{k-1}$ (note that $\rho_{1}\left(\theta^{\prime}\right)$ is a complex number!), and hence

$$
\frac{\left|\rho_{1}(\theta)\right|}{\left|\rho_{1}\left(\theta^{\prime}\right)\right|} \geq \rho_{1}(\beta) \sqrt{k-1}>1
$$

Put $\Delta_{1}:=\rho_{1}(\beta) \sqrt{k-1}$. It is clear that $\Delta_{1}$ only depends on $k$. Define $\alpha_{1}$ to be the number for which $\left(9 k^{3}\right)^{\alpha_{1}}=\Delta_{1}$ holds.

Assume $d-(\mathrm{h}+\mathrm{t}) \leq \alpha_{1} \mathrm{~h}$. By Proposition 2.2 we have

$$
M(\theta) \geq \rho_{1}(\theta)^{2 \mathrm{~h}}(k-1)^{\mathrm{h}} \geq \Delta_{1}^{2 \mathrm{~h}} \rho_{1}\left(\theta^{\prime}\right)^{2 \mathrm{~h}}(k-1)^{\mathrm{h}}=\Delta_{1}^{2 \mathrm{~h}}=\left(9 k^{3}\right)^{2 \alpha_{1} \mathrm{~h}}
$$

and

$$
M\left(\theta^{\prime}\right) \leq B \mathrm{~h}\left(9 k^{3}\right)^{\alpha_{1} \mathrm{~h}}
$$

Now it is easy to see that there exists some $R_{3}>0$ (only depending on $k$, since $\alpha_{1}$ and $B$ only depend on $k)$, so that if $\mathrm{h}>R_{3}$, then $M(\theta) \neq M\left(\theta^{\prime}\right)$ which is a contradiction.

Case 2. $\theta^{\prime} \notin[-2 \sqrt{k-1}, 2 \sqrt{k-1}]$.

Since $\theta^{\prime} \notin[-2 \sqrt{k-1}, 2 \sqrt{k-1}]$, we must have $\gamma<k-2 \sqrt{k-1}$. Without loss of generality we can assume $|\theta|>\left|\theta^{\prime}\right|$ since $\theta \geq \beta>2 \sqrt{k-1}$. Put

$$
\Delta_{2}:=\min \left\{\frac{\rho_{1}(x+\gamma)}{\rho_{1}(x)} \mid 2 \sqrt{k-1} \leq x \leq k-\gamma\right\}
$$

noting that $\Delta_{2}$ is well defined since $\rho_{1}(x) \geq \frac{1}{\sqrt{k-1}}$, and that $\Delta_{2}$ only depends on $k$ since $\gamma$ only depends on $k$. Moreover $\Delta_{2}>1$ as $\rho_{1}$ is a strictly increasing continuous function on $[2 \sqrt{k-1}, k]$. It follows that

$$
\frac{\left|\rho_{1}(\theta)\right|}{\left|\rho_{1}\left(\theta^{\prime}\right)\right|}=\frac{\rho_{1}(|\theta|)}{\rho_{1}\left(\left|\theta^{\prime}\right|\right)} \geq \min \left\{\frac{\rho_{1}(x+\gamma)}{\rho_{1}(x)} \mid 2 \sqrt{k-1} \leq x \leq k-\gamma\right\}=\Delta_{2}>1
$$

holds. Define $\alpha_{2}$ to be the number for which $\left(9 k^{3}\right)^{\alpha_{2}}=\Delta_{2}$ holds.

Assume $d-(\mathrm{h}+\mathrm{t}) \leq \alpha_{2} \mathrm{~h}$. By Proposition 2.2 we have

$$
M(\theta) \geq \rho_{1}(\theta)^{2 \mathrm{~h}}(k-1)^{\mathrm{h}} \geq \Delta_{2}^{2 \mathrm{~h}} \rho_{1}\left(\theta^{\prime}\right)^{2 \mathrm{~h}}(k-1)^{\mathrm{h}}=\rho_{1}\left(\theta^{\prime}\right)^{2 \mathrm{~h}}(k-1)^{\mathrm{h}}\left(9 k^{3}\right)^{\alpha_{2} \mathrm{~h}}
$$


and

$$
M\left(\theta^{\prime}\right) \leq A \rho_{1}\left(\theta^{\prime}\right)^{2 \mathrm{~h}}(k-1)^{\mathrm{h}}\left(9 k^{3}\right)^{\alpha_{2} \mathrm{~h}} .
$$

Now it is easy to see that there exists some $R_{4}>0$ (only depending on $k$, since $\alpha_{2}$ and $A$ only depend on $k)$, so that if $\mathrm{h}>R_{4}$, then $M(\theta) \neq M\left(\theta^{\prime}\right)$ which is a contradiction.

Claim 3 now follows by putting $\alpha:=\min \left\{\alpha_{1}, \alpha_{2}\right\}$ and $R:=\max \left\{R_{3}, R_{4}\right\}$.

Using these claims it is now straight-forward to complete the proof of the theorem. We first show that there are finitely many triangle-free distance-regular graphs $\Gamma$ with degree $k$ which have no eigenvalue in $S_{1 / 2}$. By Claim 1 , it follows that there exists some nonnegative constant $M$ depending only on $k$ so that any such $\Gamma$ must satisfy $d-(\mathrm{h}+\mathrm{t}) \leq M$. However, in [5] it is shown that there are finitely many triangle-free distance-regular graphs with degree $k$ and diameter $d$ that satisfy this last inequality.

Now, suppose that $\Gamma$ is a triangle-free distance-regular graph with degree $k$ and diameter $d$ which has some eigenvalue in $S_{1 / 2}$. Let $\alpha, R>0$ be the constants whose existence is given by Claim 3 and suppose that $d-(\mathrm{h}+\mathrm{t}) \leq \alpha \mathrm{h}$ holds. By Claim $3, \mathrm{~h}<R$ holds for any such distance-regular graph $\Gamma$. But there are finitely many such graphs since this last inequaility implies that the diameter of $\Gamma$ is bounded by a function of $k$ (which can be seen using, for example, Ivanov's bound [6, Theorem 5.9.8]). This completes the proof of the theorem.

\section{Acknowledgment}

Jack Koolen would like to thank Sasha Ivanov for his hospitality at the Imperial College of Science, London, where this work began. This work was mainly done when the first author was visiting the $\mathrm{Com}^{2} \mathrm{MaC}$ center at POSTECH, Pohang, Korea, and he thanks the $\mathrm{Com}^{2} \mathrm{MaC}-\mathrm{KOSEF}$ for its support. The second author thanks the Swedish Research council (VR) for its support.

\section{References}

1. E. Bannai and T. Ito, "The study of distance-regular graphs from the algebraic (i.e. character theoretical) viewpoint," Proceedings of Symposia in Pure Mathematics 47 (1987), 343-349.

2. E. Bannai and T. Ito, "On distance-regular graphs with fixed valency," Graphs and Combinatorics 3 (1987), 95-109.

3. E. Bannai and T. Ito, "On distance-regular graphs with fixed valency II," Graphs and Combinatorics 4 (1988), 219-228.

4. E. Bannai and T. Ito, "On distance-regular graphs with fixed valency III," Journal of Algebra 107 (1987), 43-52.

5. E. Bannai and T. Ito, "On distance-regular graphs with fixed valency IV," European Journal of Combinatorics 10 (1989), 137-148.

6. A.E. Brouwer, A.M. Cohen, and A. Neumaier, Distance-Regular Graphs, Ergebnisse der Mathematik 3.18, Springer, Heidelberg, 1989.

7. J.H. Koolen and V. Moulton, "On a conjecture of Bannai and Ito: There are finitely many distance-regular graphs with degree 5, 6 or 7," European Journal of Combinatorics 23 (2002), 987-1006.

8. P. Terwilliger, "Eigenvalue multiplicities of highly symmetric graphs," Discrete Mathematics 41 (1982), 295302. 